David Grimm

\title{
Splitting fields of conics and sums of squares of rational functions
}

\author{
Received: 3 April 2012 / Revised: 7 July 2012
}

Published online: 6 November 2012

\begin{abstract}
Given a geometrically unirational variety over an infinite base field, we show that every finite separable extension of the base field that splits the variety is the residue field of a closed point. As an application, we obtain a characterization of function fields of smooth conics in which every sum of squares is a sum of two squares.
\end{abstract}

\section{Introduction}

Let $K$ be a field. The first aim of this article is to prove the following result, which will be achieved in Theorem 2.7 .

Theorem A. Assume that $K$ is infinite. Let $L / K$ be a finite separable extension and $V$ a geometrically integral variety over $K$ such that $V_{L}$ is unirational. Then there exists a regular point $P \in V$ such that $K(P) \cong_{K} L$.

The result applies in particular to smooth conics. It was obtained as a tool to study sums of squares in their function fields. The study of sums of squares of algebraic function fields is the second main aspect of this article.

We say that $K$ is real if -1 is not a sum of squares in $K$, and nonreal otherwise. The pythagoras number of $K$, denoted $p(K)$, is defined as the smallest positive integer $n$ such that every sum of squares in $K$ is equal to a sum of $n$ squares; if no such integer exists we set $p(K)=\infty$. If $p(K)=1$ we say that $K$ is pythagorean. If $K$ is real and every finite real extension of $K$ is pythagorean, we say that $K$ is hereditarily pythagorean. Note that any field of characteristic 2 is pythagorean. In the sequel we assume that the characteristic of $K$ is different from 2 . We refer to [8, Chap. 7] and [5, Chap. XI, Sect. 6 and Chap. XIII, Sections $3 \&$ 5] for an overview of known results on pythagoras numbers of fields.

Let $F / K$ be an algebraic function field; by this we mean that $F$ is a finitely generated extension of transcendence degree one over $K$. We refer to [9, Chap. I] for the basic theory of algebraic function fields, including the definition of the genus.

Let us further assume that $K$ is relatively algebraically closed in $F$. We want to study the pythagoras number of $F$ in terms of properties of $K$ and in particular relate $p(F)$ and $p(K)$. This problem is widely open in general. In particular, it

D. Grimm ( $\varangle)$ : Ecole Polytéchnique, Fédérale de Lausanne, MA C3 1015, Lausanne, Switzerland. e-mail: david.grimm@epfl.ch

Mathematics Subject Classification (2010): 14G05, 12D15, 14H05, 14H45 
is not known whether $p(F)$ can be bounded in terms of $p(K)$. It follows from [5, Chap.VIII, 5.7] that $p(F) \geq 2$. In this article, we study the situation where $p(F)=2$. The following result will be obtained in Theorem 3.2.

Theorem B. Assume that $F / K$ has genus zero and that -1 is not a square in $K$. Then $p(F)=2$ if and only if $K$ is hereditarily pythagorean and, in case $F$ is nonreal, uniquely ordered.

Note that if -1 is a square in $K$ then $p(F)=2$. Theorem B generalizes [2, Chap. III, Thm. 4], which treats the case where $F / K$ is rational. The 'if' implication in Theorem $B$ was shown in [10, Theorems $2 \& 3$ ]. The main point here is to show the 'only if' implication.

In $[1,4.4]$ it was asked whether an algebraic function field can only have pythagoras number two if the field of constants either contains a square root of -1 or is hereditarily pythagorean. Theorem $\mathrm{B}$ gives a positive answer for algebraic function fields of genus zero. The next result, which will be obtained in Theorem 4.7, extends this to function fields of Cassels-Catalan curves.

A plane curve over $K$ defined by $1=a X^{n}+b Y^{m}$ where $n, m \in \mathbb{N}$ are prime to the characteristic of $K$ and $a, b \in K^{\times}$is called a Cassels-Catalan curve.

Theorem C. Assume that $K$ is not hereditarily pythagorean and that -1 is not a square in $K$. Assume that $F$ is the function field of a Cassels-Catalan curve over $K$. Then $p(F) \geq 3$.

The method in the proofs of Theorems $\mathrm{B}$ and $\mathrm{C}$ is to search a point on the curve having a nonreal residue field in which -1 is not a square. Given such a point, we relate a minimal representation of -1 as a sums of squares in its residue field to a sum of squares in $F$ that is not a sum of two squares, using a valuation argument formulated in Proposition 3.1. In general, finding such a point on a Cassels-Catalan curve is technical. In the special case $n=m=2$, that is, for smooth conics, Theorem A yields a more conceptual argument for the existence of such a point.

\section{Points on geometrically unirational varieties}

The main goal of this section is to prove Theorem A. I am indebted to Adrian Wadsworth for contributing a key idea to its proof.

An integral variety over a field is called unirational, if there exists a dominant morphism from an open subscheme of an affine space to the variety (or equivalently, if the function field of the variety embeds over the base field into a rational function field), and moreover rational if this morphism has an inverse that is defined on an open subscheme of the variety (or equivalently, if the function field is isomorphic over the base field to a rational function field).

Let $V$ be a $K$-vector space of dimension $n<\infty$. We call a map $V \rightarrow K$ a $K$-polynomial function if it is given by the evaluation of a polynomial in $n$ variables over $K$, after identifying $V$ with $K^{n}$ by the choice of an arbitrary basis for $V$. We call the zero locus $H(g)$ of a nonzero $K$-polynomial function $g: V \rightarrow K$ a $K$ hyperplane, and we call the coarsest topology in $V$ in which every $K$-hyperplane is 
closed, the $K$-Zariski topology. The $K$-Zariski topology on $K$ itself is the cofinite topology.

Given a $K$-hyperplane $H(g) \subset V$ and another finite dimensional vector space $W$, a map $\varphi: V \backslash H(g) \rightarrow W$ is said to be $K$-regular if there exists $r \in \mathbb{N}$ and a $K$-polynomial function $f: W \rightarrow K$, such that the map $V \backslash H(g) \rightarrow K$ given by $v \mapsto(f \circ \varphi)(v) \cdot g(v)^{r}$ extends to a $K$-polynomial function on $V$.

Given two $K$-regular maps $\varphi: V_{1} \backslash H_{1} \rightarrow V_{2}$ and $\psi: V_{2} \backslash H_{2} \rightarrow V_{3}$ with $\varphi^{-1}\left(H_{2}\right) \cup H_{1} \neq V_{1}$, the composition $\psi \circ \varphi$ is defined as a $K$-regular map on the complement of the hyperplane $\varphi^{-1}\left(H_{2}\right) \cup H_{1}$ in $V_{1}$.

Lemma 2.1. Let $L / K$ be a finite field extension. Then mult $_{L}: L \times L \rightarrow L$, $(x, y) \mapsto x y$ and $\operatorname{inv}_{L}: L \backslash\{0\} \rightarrow L, x \mapsto \frac{1}{x}$ are $K$-regular maps.

Proof. We identify $L$ with a $K$-subalgebra of $\operatorname{End}_{K}(L)$, via the algebra homomorphism that assigns to $a \in L$ the left-multiplication $x \mapsto a x$. The multiplication on $\operatorname{End}_{K}(L)$ is a $K$-regular map $\operatorname{End}_{K}(L) \times \operatorname{End}_{K}(L) \rightarrow \operatorname{End}_{K}(L)$, as can be seen by identifying End $_{K}(L)$ with a matrix algebra over $K$. Hence, its restriction mult $_{L}: L \times L \rightarrow L$ to $L$ is also a $K$-regular map. The subset of noninvertible elements of $\operatorname{End}_{K}(L)$ is a $K$-hyperplane given by a determinant polynomial. This hyperplane restricts to the single point set $\{0\}$ in $L$. The inversion map on the invertible elements is a $K$-regular map on $\operatorname{End}_{K}(L)$ by Cramer's Rule. Restricting the map to $L \backslash\{0\}$, we obtain that $\operatorname{inv}_{L}: L \backslash\{0\} \rightarrow L$ is also a $K$-regular map.

For $f \in K(t)$, choose $g, h \in K[t]$ relatively prime such that $f=\frac{g}{h}$. We write $f: K \rightarrow K$ for the regular map $K \backslash H(h) \rightarrow K$ defined by $x \mapsto \frac{g(x)}{h(x)}$.

Corollary 2.2. Let $L / K$ be a finite extension and $f \in L(t)$. The L-regular map $f: L \rightarrow L$ is a $K$-regular map.

Proof. First, we show this in the case where $f \in L[t]$. Let $s=[L: K]$ and fix an arbitrary $K$-basis $\left(\ell_{1}, \ldots, \ell_{s}\right)$ of $L$. Write $f=f_{0}+f_{1} t+\cdots+f_{d} t^{d}$ with $d \in \mathbb{N}$ and $f_{0}, \ldots, f_{d} \in L$. For $z \in L$ write $z=r_{1} \ell_{1}+\cdots+r_{s} \ell_{s}$ with $r_{1}, \ldots r_{s} \in K$. We can consider $f(z)=f\left(r_{1} \ell_{1}+\cdots+r_{s} \ell_{s}\right)$ as a polynomial function over $L$ in $s$ variables evaluated at $\left(r_{1}, \ldots, r_{s}\right)$. We can choose $\tilde{f}_{1}, \ldots, \tilde{f}_{s} \in K\left[X_{1}, \ldots, X_{s}\right]$ such that $f\left(r_{1} \ell_{1}+\cdots+r_{s} \ell_{s}\right)=\tilde{f}_{1}\left(r_{1}, \ldots, r_{s}\right) \ell_{1}+\cdots+\tilde{f}_{s}\left(r_{1}, \ldots, r_{s}\right) \ell_{s}$. Hence the map $L \rightarrow L, z \mapsto f(z)$ is given by the polynomials $\tilde{f}_{1}, \ldots, \tilde{f}_{s}$ over $K$. Now assume that $f \in L(t)$. Let $g, h \in L[t]$ be relatively prime such that $f=\frac{g}{h}$. Then the map $L \backslash H(h) \rightarrow L$ given by $z \mapsto f(z)$ is composed by $K$-regular maps

$$
f: L \backslash H(h) \stackrel{(g, h)}{\longrightarrow} L \times(L \backslash H(h)) \stackrel{\mathrm{id}_{\mathrm{L}} \times \mathrm{inv}_{\mathrm{L}}}{\longrightarrow} L \times L \stackrel{\text { mult }_{\mathrm{L}}}{\longrightarrow} L,
$$

where $(g, h)(x)=(g(x), h(x))$ and $\left(\operatorname{id}_{L} \times \operatorname{inv}_{L}\right)(x, y)=\left(x, y^{-1}\right)$.

Lemma 2.3. Let $L / K$ be a finite field extension. For every $f \in L(t)$ there exist $g \in L[t]$ and $h \in K[t]$ such that $f=\frac{g}{h}$.

Proof. Choosing $\alpha_{1}, \ldots, \alpha_{n} \in L$ such that $L=K\left[\alpha_{1}, \ldots, \alpha_{n}\right]$, we have that $L(t)=K\left[\alpha_{1}, \ldots, \alpha_{n}\right](t)=K(t)\left[\alpha_{1}, \ldots, \alpha_{n}\right]$. 
Proposition 2.4. Assume that $K$ is infinite. Let $L / K$ be a proper finite extension that is not purely inseparable. Let $f \in L(t)$ such that $f(z) \in K$ for every $z \in L$ where $f(z)$ is defined. Then $f \in K$.

Proof. We first show that $f \in K(t)$. By Lemma 2.3, there exists $g \in L[t]$ and $h \in K[t]$ such that $f=\frac{g}{h}$. Write $g=g_{0}+g_{1} t+\cdots+g_{d} t d$ with $d \in \mathbb{N}$ and $g_{0}, \ldots, g_{d} \in L$. Evaluating this polynomial in $d+1$ distinct elements $\alpha_{0}, \ldots, \alpha_{d} \in$ $K \backslash H(h)$ yields that

$$
\left(\begin{array}{cccc}
1 & \alpha_{0} & \cdots & \alpha_{0}^{d} \\
1 & \alpha_{1} & \cdots & \alpha_{1}^{d} \\
\vdots & \vdots & & \vdots \\
1 & \alpha_{d} & \cdots & \alpha_{d}^{d}
\end{array}\right) \cdot\left(\begin{array}{c}
g_{0} \\
g_{1} \\
\vdots \\
g_{d}
\end{array}\right)=\left(\begin{array}{c}
f\left(\alpha_{0}\right) h\left(\alpha_{0}\right) \\
f\left(\alpha_{1}\right) h\left(\alpha_{1}\right) \\
\vdots \\
f\left(\alpha_{d}\right) h\left(\alpha_{d}\right)
\end{array}\right) \in K^{d+1}
$$

Since the matrix on the left is invertible, we have that $g_{0}, \ldots, g_{d} \in K$. Hence $g \in K[t]$ and thus $f \in K(t)$.

In order to show that $f \in K$, we fix an element $\beta \in L \backslash K$ that is separable over $K$. Let $\sigma$ be a $K$-automorphism of the algebraic closure of $L$ such that $\sigma(\beta) \neq \beta$. Since $f(z) \in K$ for all $z \in L \backslash h^{-1}(\{0\})$, it follows that $g\left(r_{0}+r_{1} \beta\right) h\left(r_{0}+r_{1} \sigma(\beta)\right)=$ $g\left(r_{0}+r_{1} \sigma(\beta)\right) h\left(r_{0}+r_{1} \beta\right)$ for any $r_{0}, r_{1} \in K$. Since $K$ is infinite, we obtain the polynomial identity $g(X+Y \beta) h(X+Y \sigma(\beta))=g(X+Y \sigma(\beta)) h(X+Y \beta)$ in the variables $X$ and $Y$. Since the matrix

$$
\left(\begin{array}{cc}
1 & \beta \\
1 & \sigma(\beta)
\end{array}\right)
$$

is invertible, we conclude by a linear change of variables the polynomial identity $g(X) h(Y)=g(Y) h(X)$. Hence $f=\frac{g}{h} \in K$.

Proposition 2.5. Assume that $K$ is an infinite field. Let $L / K$ be a finite separable extension. Let $f \in L(t) \backslash L$. Then there exists $\alpha \in L$ such that $f(\alpha)$ is defined and $K(f(\alpha)) \cong_{K} L$.

Proof. By Corollary 2.2, the $L$-regular map $f: L \rightarrow L$ is $K$-regular. Note that the $K$-open subset in $L$ on which this regular map is defined is $K$-irreducible. As $f$ is continuous, the image of the map $f: L \rightarrow L$ is irreducible. Assume that the image of $f: L \rightarrow L$ does not contain a primitive element of $L / K$, then it is contained in the finite union of the maximal proper subfields of $L$ that contain $K$. The latter is a finite union of $K$-vector subspaces of $L$. None of those maximal proper subfields is contained in the union of the others, hence they are the $K$-irreducible components of this finite union. Thus the image of $f: L \rightarrow L$ is contained in one maximal proper subfield $E$ of $L$ containing $K$. By Proposition 2.4, we obtain that $f \in E$, which contradicts the assumption that $f \notin L$.

Remark 2.6. If $K$ is a finite field of characteristic $p$ and $L / K$ a proper finite extension, then the image of the nonconstant map $L \rightarrow L, x \mapsto x^{p}$ is a proper subfield of $L$. 
Theorem 2.7. Assume that $K$ is infinite. Let $L / K$ be a finite separable extension and $V$ be a geometrically integral variety over $K$ such that $V_{L}$ is unirational. Then the set of regular points $P \in V$ with $K(P) \cong_{K} L$ is $K$-Zariski dense in $V$.

Proof. Let $n \in \mathbb{N}$ and $U \in \mathbb{A}_{L}^{n}$ an open $L$-subvariety and $U \rightarrow V_{L}$ a dominant morphism. By [4, Chap. II, 8.16], the subset of regular points on $V$ is open dense. Hence, by the dominance of the morphism $U \rightarrow V_{L}$, we can assume $V$ to be regular affine. Let $V \hookrightarrow \mathbb{A}_{K}^{m}$ be a closed immersion for some $m \in \mathbb{N}$.

We choose a projection $\mathbb{A}_{K}^{m} \rightarrow \mathbb{A}_{K}^{1}$ such that the composition $V \hookrightarrow \mathbb{A}_{K}^{m} \rightarrow \mathbb{A}_{K}^{1}$ is not constant. Furthermore, we choose a closed immersion $\mathbb{A}_{L}^{1} \hookrightarrow \mathbb{A}_{L}^{n}$ such that preimage $W$ of $U$ with respect to this immersion is nonempty and such that $W$ is not mapped to a single point in $\mathbb{A}_{L}^{1}$. Considering the commutative diagram

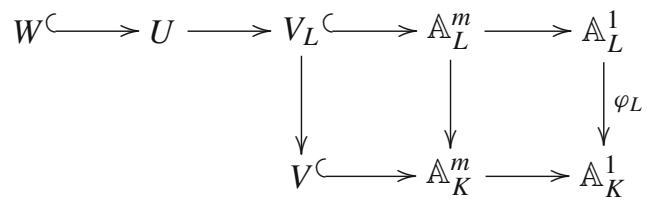

whose vertical arrows are the base-change morphisms, we see that it is sufficient to find a rational point $P \in W$ that is mapped to a closed point in $\mathbb{A}_{K}^{1}$ with residue field $L$. Equivalently, it is sufficient to find a rational point $P \in W$ whose image in $\operatorname{Spec}(L[Y])=\mathbb{A}_{L}^{1}$ corresponds to a maximal ideal generated by a linear polynomial $Y-\beta$, where $\beta \in L$ is such that $L=K(\beta)$, since the preimage of this maximal ideal under the dual homomorphism to the base change $\varphi_{L}$ will be the maximal ideal in $\operatorname{Spec}(K[Y])=\mathbb{A}_{K}^{1}$ generated by the minimal polynomial for $\beta$ over $K$.

By shrinking $W$ if necessary, we can assume that $W=\operatorname{Spec}\left(L[X]_{h}\right)$, that is, $W$ is a principal open subscheme of $\mathbb{A}_{L}^{1}=\operatorname{Spec}(L[X])$ given by the localization of $L[X]$ after the multiplicative set $\left\{h^{i} \mid i \in \mathbb{N}_{0}\right\}$ for some $h \in L[X]$. The nonconstant map $W \rightarrow \mathbb{A}_{L}^{1}$ corresponds to a $L$-algebra homomorphism

$$
\psi: L[Y] \rightarrow L[X]_{h}
$$

with $\psi(Y) \notin L$. Say $\psi(Y)=\frac{g}{h^{r}}$ for some $r \in \mathbb{N}$ and $g \in L[X]$. For arbitrary $\alpha \in L$ such that $h(\alpha) \neq 0$, consider a maximal ideal in $L[X]_{h}$ containing $\frac{g}{h^{r}}-\frac{g(\alpha)}{h(\alpha)}$. Its inverse image in $L[Y]$ is obviously the maximal ideal generated by $Y-\frac{g(\alpha)}{h(\alpha)}$. By Proposition 2.5, there exists $\alpha \in L$ with $h(\alpha) \neq 0$ and $L=K\left(\frac{g(\alpha)}{h(\alpha)}\right)$. This shows the existence of a regular point $P \in V$ with $K(P) \cong_{K} L$. Let $V^{\prime}$ denote the complement in $V$ of the closure of the set of regular points $P \in V$ with $K(P) \cong_{K} L$. If $V^{\prime}$ is nonempty then $V_{L}^{\prime}$ is unirational and we obtain a contradiction to the first part applied to $V^{\prime}$. Hence, the set of regular points $P \in V$ with $K(P) \cong_{K} L$ is dense in $V$.

It is not clear whether Theorem 2.7 can be extended to hold for projective varieties over finite fields. In fact, already the following rational case is unknown.

Question 2.8. Let $V$ be a rational projective variety over a finite field $K$ and $L / K$ be a finite extension. Is there a point $P \in V$ such that $K(P) \cong_{K} L$ ? 


\section{Sums of squares in function fields of conics}

The following observation will allow us to show that the field of constants of certain function fields of pyhagoras number two only allow finite nonreal extensions in which -1 becomes a square or, equivalently, only allow finite real extensions that are pythagorean.

Proposition 3.1. Assume that $K$ carries a valuation with value group $\mathbb{Z}$ and with nonreal residue field $\kappa$ of characteristic different from 2 . Let $s$ be the smallest positive integer such that -1 is a sum of squares in $\kappa$. Then $p(K)>s$.

Proof. Let $v$ denote the valuation. By the choice of $s$, there exist $x_{0}, \ldots, x_{s} \in K$ with $v\left(x_{0}\right)=\ldots=v\left(x_{s}\right)=0$ and $v\left(x_{0}^{2}+\ldots+x_{s}^{2}\right)>0$. If $v\left(x_{0}^{2}+\ldots+x_{s}^{2}\right)>1$ we replace $x_{0}$ by $x_{0}+t$ for any $t \in K$ with $v(t)=1$. Hence, we may assume that $v\left(x_{0}^{2}+\ldots+x_{s}^{2}\right)=1$. We claim that $x_{0}^{2}+\ldots+x_{s}^{2}$ is not a sum of $s$ squares in $K$. Suppose on the contrary that there exist $y_{1}, \ldots, y_{s} \in K$ with $y_{1}^{2}+\ldots+$ $y_{s}^{2}=x_{0}^{2}+\ldots+x_{s}^{2}$. We can assume that $v\left(y_{1}\right) \leq v\left(y_{2}\right) \leq \ldots \leq v\left(y_{s}\right)$. Then $v\left(1+\left(y_{1}^{-1} y_{2}\right)^{2}+\ldots+\left(y_{1}^{-1} y_{s}\right)^{2}\right)>0$, and we obtain a representation of -1 as a sum of $s-1$ squares in $\kappa$, contradicting the choice of $s$.

We recall from $[3,1.3 .2 \& 1.3 .5]$ that every regular conic over a field of characteristic different from 2 is a generic splitting variety for a quaternion algebra, that is, the base field extensions that split the quaternion algebra are exactly those over which the conic becomes rational.

Theorem 3.2. Assume that $K$ is not hereditarily pythagorean and that -1 is not a square in $K$. Let $F$ be the function field of a regular conic over $K$. Then $p(F) \geq 3$.

Proof. We shall first observe that there exists a finite separable nonreal extension $K^{\prime \prime}$ of $K$ such that -1 is a sum of two squares in $K^{\prime \prime}$. In the case where $K$ is real, let $K^{\prime}$ denote a finite real extension of $K$ that is not pythagorean. In the case where $K$ is nonreal, set $K^{\prime}=K$. In both cases, there exists a sum of two squares $\sigma$ in $K^{\prime}$ that is not a square, and we set $K^{\prime \prime}=K^{\prime}(\sqrt{-\sigma})$, which is a finite separable extension of $K$.

Now consider any maximal algebraic field extension $M / K^{\prime \prime}$ in which -1 is not a square. By [5, Chap. III, 2.8], every quaternion algebra over $M$ is split, whereby every conic over $M$ is rational. The conic of the statement already splits over some finite nonreal extension $L / K$ contained in $M$. Note that -1 is not a square in $L$. In the case where $K$ is real, it is clear that $L / K$ is separable, hence there exists a point on the conic with residue field $L$ by Theorem 2.7. In the case where $K$ is nonreal, there exists a point on the conic whose residue field $L^{\prime}$ is a subfield of $L$ and contains $K$. Hence, $L$ is nonreal and -1 is not a square in $L$. In both cases Proposition 3.1 yields $p(F)>2$.

Recall that function fields of regular conics are exactly the algebraic function fields of genus zero, by [7, 7.4.1] and [9, Chap. V, 3.3]. The following corollary is Theorem B of the introduction. 
Corollary 3.3. Assume that -1 is not a square in $K$. Let $F / K$ be an algebraic function field of genus zero.

(a) Assume that $F$ is real. Then $p(F)=2$ if and only if $K$ is hereditarily pythagorean.

(b) Assume that $F$ is nonreal. Then $p(F)=2$ if and only if $K$ is hereditarily pythagorean and uniquely ordered.

Proof. A real algebraic function field of genus zero over a hereditarily pythagorean field has pythagoras number 2 , e.g. by $[1,4.10]$. The converse implication follows with Theorem 3.2. This shows $(a)$. Now assume that $K$ is hereditarily pythagorean and uniquely ordered. By [2, Chap. III, Lemma 5], a uniquely ordered hereditarily pythagorean field is a so called hereditarily euclidean field. Hence every algebraic function field has pythagoras number 2, by [1, 4.6]. Assume conversely that $p(F)=2$ for a nonreal algebraic function field of genus zero. It follows by Theorem 3.2 that $K$ is hereditarily pythagorean. Hence, $F$ is isomorphic to the function field of the conic $X^{2}+Y^{2}+Z^{2}=0$. By [1, 4.7], it follows that $K$ is uniquely ordered. This shows $(b)$.

\section{Sums of squares in function fields of Cassels-Catalan curves}

We denote by $K^{\times 2}$ the set of nonzero squares in a field $K$, and by $\pm K^{\times 2}$ we denote the set $K^{\times 2} \cup-K^{\times 2}$. The algebraic closure of $K$ is denoted $K_{\text {alg }}$.

Proposition 4.1. Assume that $K$ is infinite. Let $L$ be a finite separable non-pythagorean extension of $K$. Then there exists $\xi \in L$ such that $L=K\left(\xi^{2}\right)$ and $\xi^{2}+1 \notin L^{\times 2}$. Moreover, there exists $\sigma \in \sum L^{2} \backslash L^{\times 2}$ such that $L=K(\sigma)$ and $\sigma+1 \notin L^{\times 2}$.

Proof. Fix $z \in L$ with $z^{2}+1 \notin L^{\times 2}$. For arbitrary $v \in L^{\times}$, consider the terms $\alpha=\frac{v^{2}}{z^{2}}, \beta=v^{2}+z^{2}, \gamma=\frac{\left(z^{2}+1\right)^{2}}{v^{2}}+z^{2}, \delta=\frac{\left(z^{2}+1\right)^{2}}{z^{2} v^{2}}$, and $\epsilon=\frac{z^{2}+1}{v^{2}}$. These terms are rational functions in $v$ over $L$. Let $\mathcal{G}=\{x \in L \mid K(x)=L\}$. This is a $K$-Zariski open subset of $L$ as it is the complement of the finitely many subspaces of $L$ that correspond to the finitely many intermediate extensions of $L / K$. By Proposition 2.5 the preimage of $\mathcal{G}$ under any rational function from $L(t) \backslash L$ is nonempty. Moreover, $\mathcal{G}$ is $K$-open in $L$. As the intersection of finitely many nonempty $K$-open subsets of $L$ is nonempty, there exists $v \in L^{\times}$, such that $\alpha, \beta, \gamma, \delta, \epsilon \in \mathcal{G}$. Note that $\epsilon, \frac{1}{\epsilon} \in \sum L^{2} \backslash L^{\times 2}$. If $\epsilon+1 \notin L^{\times 2}$ we set $\sigma=\epsilon$. Otherwise we have $\frac{1+\epsilon}{\epsilon}=\frac{1}{\epsilon}+1 \notin L^{\times 2}$ and set $\sigma=\frac{1}{\epsilon}$.

Note also that $\alpha \in L^{\times 2}$ and if $\alpha+1 \notin L^{\times 2}$, choose $\xi=\frac{v}{z}$. Assume now that $\alpha+1 \in L^{\times 2}$. Then $\beta \in L^{\times 2}$. If $\beta+1 \notin L^{\times 2}$ choose $\xi \in L$ such that $\xi^{2}=\beta$. Assume now that $\beta+1 \in L^{\times 2}$. Then $v^{2}+z^{2}+1 \in L^{\times 2}$ and $v^{2}+z^{2} \in L^{\times 2}$. It follows that $\frac{\left(z^{2}+1\right)^{2}}{v^{2}}+z^{2}+1 \notin L^{\times 2}$ since $z^{2}+1 \notin L^{\times 2}$. Remember that $\delta=\frac{\left(z^{2}+1\right)^{2}}{z^{2} v^{2}}$. If $\delta+1 \notin L^{\times 2}$, choose $\xi=\frac{z^{2}+1}{z v}$. Otherwise, if $\delta+1 \in L^{\times 2}$, then $\gamma \in L^{\times 2}$ and $\gamma+1 \notin L^{\times 2}$ and we choose $\xi \in L$ such that $\xi^{2}=\gamma$ in this last case.

Lemma 4.2. Let $u \in K^{\times} \backslash \pm K^{\times 2}$ and $r \geq 1$. Let $\gamma \in K_{\text {alg }}$ be such that $\gamma^{2^{r}}=u$. Then $K^{\times} \cap K(\gamma)^{\times 2}=K^{\times 2} \cup u K^{\times 2}$. 
Proof. As $-u \notin K^{\times 2}$, and thus $-u \notin 4 K^{\times 4}$, the polynomial $T^{2^{r}}-u$ is irreducible by [6, Chap. VI, 9.1]. Write $d=\gamma^{2}, L=K(d)$ and $M=K(\gamma)$. Note that $M / L$ is a quadratic extension. As $T^{2^{r-1}} u$ is the minimal polynomial of $d$ over $K$, the norm of $d$ with respect to $L / K$ is $\pm u$. As $u \notin \pm K^{\times 2}$, it follows that $K^{\times} \cap d L^{\times 2}=\emptyset$. As $L^{\times} \cap M^{\times 2}=L^{\times 2} \cup d L^{\times 2}$, we have that

$$
K^{\times} \cap M^{\times 2}=K^{\times} \cap\left(L^{\times 2} \cup d L^{\times 2}\right)=K^{\times} \cap L^{\times 2} .
$$

The statement thus follows by induction on $r$.

Corollary 4.3. Suppose $-1 \notin K^{\times 2}$. Let $u \in K^{\times} \backslash \pm K^{\times 2}$ and $n \in \mathbb{N}$. There exists $x \in K_{\text {alg }}$ with $x^{n}=u$ and $K^{\times} \cap K(x)^{\times 2} \subseteq K^{\times 2} \cup u K^{\times 2}$.

Proof. If $n$ is odd, we can choose $x \in K_{\text {alg }}$ with $x^{n}=u$ such that $[K(x): K]$ is odd, whereby $K^{\times} \cap K(x)^{\times 2} \subseteq K^{\times 2}$. Assume now that $n$ is even. If $u \notin K^{\times 2}$, then we write $n=2^{r} m$ with $m$ odd and $r \geq 1$, and apply Lemma 4.2 together with the previous case.

Corollary 4.4. Suppose $-1 \notin K^{\times 2}$. Let $v \in K^{\times} \backslash-K^{\times 2}$ and $m \in \mathbb{N}$. There exists $y \in K_{\text {alg }}$ such that $y^{m}=v$ and $-1 \notin K(y)^{\times 2}$.

Proof. Let $r \in \mathbb{N}$ be maximal such that $2^{r} \mid m$ and $v \in K^{\times 2^{r}}$. Let $u \in K$ be such that $u^{2^{r}}=v$. We write $m=n 2^{r}$. If $n$ is odd, then we can choose $y \in K_{\text {alg }}$ such that $y^{m}=v$ and $[K(y): K]$ is odd, and it follows trivially that $-1 \notin K(y)^{\times 2}$.

Assume that $n$ is even. Then $u \notin K^{\times 2}$ by the maximality of $r$. Furthermore, we claim that $u \notin-K^{\times 2}$. If $r=0$ we have that $u=v \notin \pm K^{\times 2}$. If $r>0$ then $u \notin-K^{\times 2}$ by the maximality of $r$ and the fact that $(-u)^{2^{r}}=v$. Using Corollary 4.3, we choose $y \in K_{\text {alg }}$ such that $y^{n}=u$ and $K^{\times} \cap K(y)^{\times 2} \subseteq K^{\times 2} \cup u K^{\times 2}$. Then $y^{m}=v$ and $-1 \notin K(y)^{\times 2}$, since $u \notin-K^{\times 2}$.

Proposition 4.5. Suppose $-1 \notin K^{\times 2}$. Let $u \in K^{\times} \backslash \pm K^{\times 2}$ and $v \in K^{\times} \backslash\left(-K^{\times 2} \cup\right.$ $\left.-u K^{\times 2}\right)$. Let $n, m \geq 1$. Then there exists a finite extension $M / K$ such that $-1 \notin$ $M^{\times 2}$, with $x, y \in M$ such that $x^{n}=u$ and $y^{m}=v$, and such that $M=K(x, y)$.

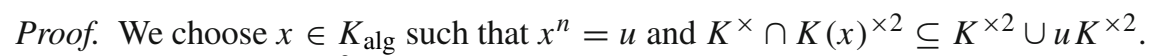
Then $-1,-v \notin K(x)^{\times 2}$. By Corollary 4.4 there exists $y \in K_{\text {alg }}$ such that $y^{m}=v$ and $-1 \notin K(x, y)^{\times 2}$. Set $M=K(x, y)$.

Corollary 4.6. Let $L / K$ be a finite field extension such that $L$ is real and not pythagorean. Let $a, b \in K$ such that $a, b \in L^{\times 2} \cup-L^{\times 2}$. For integers $n, m \geq 1$, there exists a finite extension $M / L$, such that $-1 \notin M^{\times 2}$, and with $x, y \in M$ such that $1=a x^{n}+b y^{m}$ and $M=K(x, y)$. Moreover, if $n$ or $m$ is even, we can choose $M$ to be nonreal.

Proof. By Proposition 4.1 there exists $\xi \in L$ with $\xi^{2}+1 \in \sum L^{\times 2} \backslash L^{\times 2}$ and $L=$ $K\left(\xi^{2}\right)$, and further $\sigma \in \sum L^{\times 2} \backslash L^{\times 2}$ with $L=K(\sigma)$ and $\sigma+1 \in \sum L^{\times 2} \backslash L^{\times 2}$.

In the case where $a, b \in L^{\times 2}$, set $u=-\frac{1}{a \sigma}$ and $v=\frac{1}{b}\left(1+\frac{1}{\sigma}\right)$. Then $u \notin \pm L^{\times 2}$ and $-v \notin L^{\times 2} \cup u L^{\times 2}$, as $-u v=\frac{1}{a b} \frac{\sigma+1}{\sigma^{2}}$. Moreover, $1=a u+b v$. 
In the case where $-a,-b \in L^{\times 2}$, set $u=\frac{\xi^{2}+1}{a}$ and $v=\frac{-\xi^{2}}{b}$. Then $u \notin \pm L^{\times 2}$ and $-v \notin L^{\times 2} \cup u L^{\times 2}$. Moreover, $1=a u+b v$.

In the case where $-a, b \in L^{\times 2}$ set $u=\frac{\sigma+1}{a}$ and $v=\frac{-\sigma}{b}$. Then $u \notin \pm L^{\times 2}$ and $-v \notin L^{\times 2} \cup u L^{\times 2}$. Moreover, $1=a u+b v$.

In the case where $a,-b \in L^{\times 2}$ set $u=\frac{-\sigma}{a}$ and $v=\frac{\sigma+1}{b}$. Then $u \notin \pm L^{\times 2}$ and $-v \notin L^{\times 2} \cup u L^{\times 2}$. Moreover, $1=a u+b v$.

In each case, by Proposition 4.5, there exist $x, y \in L_{\text {alg }}$ such that $x^{n}=u$ and $y^{m}=v$ and $\sqrt{-1} \notin L(x, y)$. Moreover, since $u \in L(x, y)$ and $K(u)=L$, it follows that $L(x, y)=K(x, y)$. Obviously $1=a x^{n}+b y^{m}$ as $1=a u+b v$. Set $M=L(x, y)$. Now suppose that $n$ or $m$ is even. By symmetry, we can assume that $n$ is even. Then $x^{n}=u$ is both a square in $M$ and, by the choices of $u$ in each case, a negative sum of squares in $M$. Thus $M$ is not real.

Note that Cassels-Catalan curves are smooth. In particular, the local ring of any closed point is a discrete valuation ring. We now prove Theorem $\mathrm{C}$ of the introduction.

Theorem 4.7. Assume that $K$ is not hereditarily pythagorean and that -1 is not a square in $K$. Let $F$ be the function field of a Cassels-Catalan curve over $K$. Then $p(F) \geq 3$.

Proof. Assume that $F$ is the function field of the curve $1=a X^{n}+b Y^{m}$ for some $a, b \in K^{\times}$and $n, m \geq 1$ prime to the characteristic of $K$. Assume first that $K$ is nonreal. If $-a$ is not a square in $K$, choose $x \in K_{\text {alg }}$ such that $x^{n}=\frac{1}{a}$ and $\sqrt{-1} \notin K(x)$ as in Corollary 4.4. Then $P=(x, 0)$ is a point on the curve and -1 is not a square in $K(P)$. If $-b$ is not a square in $K$ we can proceed analogous. So we assume that both $-a$ and $-b$ are not squares in $K$. Choose $z \in K$ such that $z^{2}+1$ is not a square in $K$. Choose again $x \in K_{\text {alg }}$ such that $x^{n}=\frac{z^{2}}{-a}$ and -1 is not a square in $K(x)$. Then $\frac{1}{b}$ is not a square in $K(x)$ and we also find some $y \in K_{\text {alg }}$ such that $y^{m}=\frac{-1}{b}$ and that -1 is not a square in $K(x, y)$, as in Proposition 4.5. Again $P$ is a point on the curve for which -1 is not a square in $K(P)$. In either case, we obtain that $p(F) \geq 3$ by Proposition 3.1 in the case where $K$ is not real.

Now we assume that $K$ is real. Let us first consider the case where $n$ is odd. Then $F$ is clearly an odd degree extension of the rational algebraic function field $K(X)$. Then $3 \leq p(K(X)) \leq p(F)$ by Springer's Theorem [5, Chap. VII, 2.7] and Corollary 3.3. Hence we assume that $n$ is even. Suppose there exists a finite real extension $L / K$ that is not pythagorean. We can assume that $a$ or $-a$, as well as one of $b$ or $-b$ is a square in $L$, since we can replace $L$ by one of the four extensions $L(\sqrt{ \pm a})(\sqrt{ \pm b})$ if necessary; note that none of those extensions is pythagorean, by [5, Chap. VIII, 5.7], and at least one of them is real. By Corollary 4.6 there exists a point $P$ on the curve such that $K(P)$ is nonreal and -1 is not a square in $K(P)$. Again, Proposition 3.1 yields that $p(F) \geq 3$.

In [1, 4.3], it was shown that $p(F) \geq 3$ for any algebraic function field $F / K$ having divisors of odd degree when $K$ is not hereditarily pythagorean and -1 is not a square in $F$. For $a, b \in K^{\times}$such that the conic $1=a X^{2}+b Y^{2}$ is not split, 
the Cassels-Catalan curve $1=a X^{n}+b Y^{m}$ with $n, m$ even has no divisor of odd degree.

Acknowledgements. This work is part of my Ph.D. thesis at Universität Konstanz. I am grateful to my advisor Karim Johannes Becher for his support. Part of the research was made during a research stay with Kevin Hutchinson at University College Dublin. I am further grateful to David Leep, Jan Van Geel and Adrian Wadsworth for taking interest in my work and for helpful discussions. Financial support was received from the Deutsche Forschungsgemeinschaft (Project Quadratic Forms and Invariants, BE2614/3), from the Science Foundation of Ireland Research Frontiers Programme (Grant 05/RFP/MAT0022) and from the Swiss National Science Foundation (Grant 200020-124785/1).

\section{References}

[1] Becher, K.J., Van Geel, J.: Sums of squares in function fields of hyperelliptic curves. Math.Z. 261, 829-844 (2009)

[2] Becker, E.: Hereditarily-pythagorean fields and orderings of higher level, Monografias de Mathematica 29, Rio de Janeiro (1978)

[3] Gille, P., Szamuely, T.: Central simple algebras and Galois cohomology. Cambridge studies in advanced mathematics, vol. 101, xi, 343 p. Cambridge University Press, Cambridge (2006)

[4] Hartshorne, R.: Algebraic geometry. Graduate texts in mathematics, vol. 52. Springer, New York (1983)

[5] Lam, T.Y.: Introduction to quadratic forms over fields. Graduate studies in mathematics, vol. 67, AMS, Rhode Island (2004)

[6] Lang, S.: Algebra - revised third edition. Graduate texts in mathematics, vol. 211. Springer, New York, (2002)

[7] Liu, Q.: Algebraic geometry and arithmetic curves. Oxford graduate texts in mathematics, vol. 6. Oxford University Press, Oxford (2002)

[8] Pfister, A.: Quadratic forms with applications to algebraic geometry and topology, vol. 217. Mathematical Society Lecture Note Series, London (1995)

[9] Stichtenoth, H.: Algebraic function fields and codes. 2nd ed. Graduate texts in mathematics, vol. 254. Springer, Berlin (2009)

[10] Tikhonov, S.V., Yanchevskiŭ, V.I.: Pythagoras numbers of function fields of regular conics over hereditarily pythagorean fields. Dokl. Nats. Akad. Nauk Belarusi 47(2), $5-8(2003)$ 